\title{
A DUAL MINKOWSKI TYPE INEQUALITY
}

\section{YAN WANG AND GUANGTING WANG}

Abstract. The Orlicz Brunn-Minkowski theory was developed by Lutwak, Yang and Zhang. It is an extension of the $L_{p}$ Brunn-Minkowski theory. In this paper, a new function on the set of star bodies is introduced. A dual Minkowski type inequality about this function is established. Some properties of the new function are presented.

Mathematics subject classification (2010): 52A20, 52A40.

Keywords and phrases: Star body, dual Minkowski type inequality, harmonic combination.

\section{REFERENCES}

[1] R. J. GARDNER, The Brunn-Minkowski inequality, Bull. Amer. Math. Soc., 39, (2002), 355-405.

[2] R. J. Gardner, Geometric Tomography, 2nd edition, Encyclopedia Math. Appl., 58, Cambridge University Press, Cambridge, 2006.

[3] R. J. Gardner, D. Hug, W. Weil, The Orlicz-Brunn-Minkowski theory: A general framework, additions, and inequalities, J. Differential Geom., 97, (2014), 427-476.

[4] P. M. Gruber, Convex and Discrete Geometry, Grundlehren Math. Wiss., 336, Springer, Berlin, 2007.

[5] C. HaberL, Lp intersection bodies, Adv. Math., 217, (2008), 2559-2624.

[6] C. Haberl, E. Lutwak, D. Yang, G. Zhang, The even Orlicz Minkowski problem, Adv. Math., 224, (2010), 2485-2510.

[7] C. Haberl, F. Schuster, J. Xiao, An asymmetric affine Pólya-Szegö principle, Ann. of Math., 352, (2012), 517-542.

[8] C. Haberl, F. Schuster, Asymmetric affine $L_{p}$ Sobolev inequalities, J. Funct. Anal., 257, (2009), 641-658.

[9] C. Haberl, F. Schuster, General $L_{p}$ affine isoperimetric inequalities, J. Differential Geom., 83, (2009), 1-26.

[10] G. Hansen, H. Martini, Dispensable points, radial functions, and boundaries of starshaped sets, Acta Sci. Math. (Szeged), 80, (2014), 689-699.

[11] G. H. Hardy, J. E. Littlewood, G. Pólya, Inequalities, Cambridge Univ. Press, Cambridge, 1934.

[12] Q. Huang, B. He, On the Orlicz Minkowski problem for polytopes, Discrete Comput. Geom., 48, (2012), 281-297.

[13] A. LI, G. Leng, A new proof of the Orlicz Busemann-Petty centroid inequality, Proc. Amer. Math. Soc., 139, (2011), 1473-1481.

[14] M. Ludwig, Minkowski valuations, Trans. Amer. Math. Soc., 357, (2005), 4191-4213.

[15] M. Ludwig, General affine surface areas, Adv. Math., 224, (2010), 2346-2360.

[16] M. Ludwig AND M. ReITZNER, A classification of $S L(n)$ invariant valuations, Ann. of Math., 172, (2010), 1223-1271.

[17] E. LutwaK, The Brunn-Minkowski-Firey theory. I. Mixed volumes and the Minkowski problem, J. Differential Geom., 38, (1993), 131-150.

[18] E. LutwaK, The Brunn-Minkowski-Firey theory. II. Affine and geominial surface areas, Adv. Math., 118, (1996), 244-294.

[19] E. LutwaK, D. YAng, G. ZhANG, $L_{p}$ affine isoperimetric inequalities, J. Differential Geom., 56, (2000), 111-132. 
[20] E. Lutwak, D. Yang, G. Zhang, Sharp affine $L_{p}$ Sobolev inequalities, J. Differential Geom., 62, (2002), 17-38.

[21] E. LutwaK, D. YAng, G. Zhang, Volume inequalities for subspaces of $L_{p}$, J. Differential Geom., 68, (2004), 159-184.

[22] E. Lutwak, D. Yang, G. Zhang, Olicz projection bodies, Adv. Math., 223, (2010), 220-242.

[23] E. Lutwak, D. YAng, G. Zhang, Olicz centroid bodies, J. Differential Geom., 84, (2010), 365387.

[24] M. Meyer and E. Werner, On the p-affine surface area, Adv. Math., 152, (2000), 288-313.

[25] L. Parapatits, $S L(n)$-contravariant $L_{p}$-Minkowski valuations, Trans. Amer. Math. Soc., 366, (2014), 1195-1211.

[26] L. Parapatits, $S L(n)$-covariant $L_{p}$-Minkowski valuations, J. Lond. Math. Soc., 89, (2014), 397414.

[27] R. SCHNEIDER, Convex bodies: the Brunn-Minkowski theory, second edition, Cambridge University Press, Cambridge, 2014.

[28] D. XI, H. Jin, And G. Leng, The Orlicz Brunn-Minkowski inequality, Adv. Math., 260, (2014), $350-374$.

[29] D. Zou, G. XIONG, Orlicz-John ellipsoids, Adv. Math., 265, (2014), 132-168. 